TRANSACTIONS OF THE

AMERICAN MATHEMATICAL SOCIETY

Volume 362, Number 3, March 2010, Pages 1491-1503

S 0002-9947(09)04795-3

Article electronically published on July 17, 2009

\title{
HAMILTONIAN STATIONARY CONES AND SELF-SIMILAR SOLUTIONS IN HIGHER DIMENSION
}

\author{
YNG-ING LEE AND MU-TAO WANG
}

\begin{abstract}
In an upcoming paper by Lee and Wang, we construct examples of two-dimensional Hamiltonian stationary self-shrinkers and self-expanders for Lagrangian mean curvature flows, which are asymptotic to the union of two Schoen-Wolfson cones. These self-shrinkers and self-expanders can be glued together to yield solutions of the Brakke flow - a weak formulation of the mean curvature flow. Moreover, there is no mass loss along the Brakke flow. In this paper, we generalize these results to higher dimensions. We construct new higher-dimensional Hamiltonian stationary cones of different topology as generalizations of the Schoen-Wolfson cones. Hamiltonian stationary self-shrinkers and self-expanders that are asymptotic to these Hamiltonian stationary cones are constructed as well. They can also be glued together to produce eternal solutions of the Brakke flow without mass loss. Finally, we show that the same conclusion holds for those Lagrangian self-similar examples recently found by Joyce, Tsui and the first author.
\end{abstract}

\section{INTRODUCTION}

The existence of special Lagrangians in Calabi-Yau manifolds recently received much attention due to the critical role they play in the T-duality formulation of Mirror symmetry of Strominger-Yau-Zaslow 14. Special Lagrangians are calibrated submanifolds and thus are volume minimizers [3]. One potential approach to the construction of special Lagrangians is the mean curvature flow - the negative gradient flow of the volume functional. However, the long-time existence of such flows can only be verified in some special cases; see for example [11, 12, 15, and [16. In this article, we construct special weak solutions of the Lagrangian mean curvature flows.

Our ambient space is always the complex Euclidean space $\mathbb{C}^{n}$ with coordinates $z^{i}=x^{i}+\sqrt{-1} y^{i}$, the standard symplectic form $\omega=\sum_{i=1}^{n} d x^{i} \wedge d y^{i}$, and the standard almost complex structure $J$ with $J\left(\frac{\partial}{\partial x^{i}}\right)=\frac{\partial}{\partial y^{i}}$. A Lagrangian submanifold is an $n$-dimensional submanifold in $\mathbb{C}^{n}$ on which the symplectic form $\omega$ vanishes. On a

Received by the editors February 12, 2008.

2000 Mathematics Subject Classification. Primary 53C44, 53D12; Secondary 58J35.

Key words and phrases. Hamiltonian stationary, Lagrangian mean curvature flow, selfshrinker, self-expander, eternal solution, Brakke flow.

The first author would like to thank R. Schoen for helpful discussions and hospitality during her visit at Stanford University. The first author was supported by Taiwan NSC grant 96-2628-M-002.

The second author wishes to thank the support of the Taida Institute for Mathematical Sciences during the preparation of this article. The second author was supported by NSF grant DMS0605115 and a Sloan research fellowship. 
Lagrangian submanifold $L$ the mean curvature vector $H$ is given by

$$
H=J \nabla \theta,
$$

where $\theta$ is the Lagrangian angle and $\nabla$ is the gradient on $L$. The Lagrangian angle $\theta$ can be defined by the relation that

$$
*_{L}\left(d z^{1} \wedge \cdots \wedge d z^{n}\right)=e^{i \theta},
$$

where $*_{L}$ is the Hodge ${ }^{*}$-star operator on $L$. We recall

Definition 1. A Lagrangian submanifold $L$ is called Hamiltonian stationary if the Lagrangian angle is harmonic; i.e. $\Delta \theta=0$, where $\Delta$ is the Laplace operator on $L$. $L$ is a special Lagrangian if $\theta$ is a constant function.

A Hamiltonian stationary Lagrangian submanifold is a critical point of the volume functional among all Hamiltonian deformations, and a special Lagrangian is a volume minimizer in its homology class.

By the first variation formula, the mean curvature vector points to the direction where the volume is most rapidly decreased. As the special Lagrangians are volume minimizers, it is thus natural to use the mean curvature flow in the construction of special Lagrangians. Equation (1.1) implies that the mean curvature flow is a Lagrangian deformation, i.e. a Lagrangian submanifold remains Lagrangian along the mean curvature flow. In a geometric flow, the singularity often models on a soliton solution. In the case of mean curvature flows, one type of soliton solution of particular interest is the one moved by scaling in the Euclidean space. We recall:

Definition 2. A submanifold of the Euclidean space is called a self-similar solution if

$$
F^{\perp}=2 c H
$$

for some nonzero constant $c$, where $F^{\perp}$ is the normal projection of the position vector $F$ in the Euclidean space and $H$ is the mean curvature vector. It is called a self-shrinker if $c<0$ and a self-expander if $c>0$.

It is not hard to see that if $F$ is a self-similar solution, then $F_{t}$ defined by $F_{t}=$ $\sqrt{\frac{t}{c}} F$ is moved by the mean curvature flow. By Huisken's monotonicity formula [6], any central blow up of a finite-time singularity of the mean curvature flow is a self-similar solution. In this article, we obtain higher-dimensional Hamiltonian stationary cones with different topology as generalizations of the Schoen-Wolfson cones. We also obtain Hamiltonian stationary self-shrinkers and self-expanders which are asymptotic to these cones. Altogether they form solutions of the Brakke flow (see 3.1), which is a weak formulation of the mean curvature flow proposed by Brakke in [1]. To be more precise, we prove:

Theorem 1.1. Assume that $\lambda_{j}>0$ for $1 \leq j \leq k<n$ and $\lambda_{j}<0$ for $k<j \leq n$ are integers satisfying $\sum_{j=1}^{n} \lambda_{j}>0$. Define

$$
V_{t}=\left\{\left(x_{1} e^{i \lambda_{1} s}, \cdots, x_{n} e^{i \lambda_{n} s}\right): 0 \leq s<\pi, \sum_{j=1}^{n} \lambda_{j} x_{j}^{2}=(-2 t) \sum_{j=1}^{n} \lambda_{j},\left(x_{1}, \cdots, x_{n}\right) \in \mathbb{R}^{n}\right\} .
$$

Then $V_{t}$ is Hamiltonian stationary. It is a self-shrinker for $t<0$, a self-expander for $t>0$, and a cone for $t=0$. Moreover, the varifold $\bigcup_{t} V_{t},-\infty<t<\infty$, forms an eternal solution for Brakke flow without mass loss. 
Our construction of Lagrangian self-similar solutions is generalized to the nonHamiltonian stationary case by Joyce, Tsui and the first author in [9]. These examples can also be glued together to yield eternal solutions of the Brakke flow without mass loss.

Theorem 1.2. Let $k$ be a positive integer less than $n$. Given $\lambda_{j}>0$ for $1 \leq j \leq$ $k<n$ and $\lambda_{j}<0$ for $k<j \leq n$, let $w_{1}(s), \cdots, w_{n}(s): \mathbb{R} \rightarrow \mathbb{C} \backslash\{0\}$ be those periodic functions with period $T$, which are obtained in Theorem $\mathrm{F}$ in [9] with $\alpha=1$. Define

$$
V_{t}=\left\{\left(x_{1} w_{1}(s), \cdots, x_{n} w_{n}(s)\right): 0 \leq s<T, \sum_{j=1}^{n} \lambda_{j} x_{j}^{2}=2 t,\left(x_{1}, \cdots, x_{n}\right) \in \mathbb{R}^{n}\right\} .
$$

Then $V_{t}$ is a Lagrangian self-shrinker for $t<0$, a Lagrangian self-expander for $t>0$, and a Lagrangian cone for $t=0$. Moreover, the varifold $\bigcup_{t} V_{t},-\infty<t<\infty$, forms an eternal solution for Brakke flow without mass loss.

The choice of $\alpha=1$ in Theorem 1.2 is arbitrary; there is a lot of freedom to rescale the constants as discussed in [9, Remark 3.2]. Indeed, choosing $\alpha=-1$ instead will perhaps make the statement more consistent with Theorem 1.1. When $\lambda_{j}$ are all positive, our construction gives Hamiltonian stationary self-shrinkers $V_{t}$ for $t<0$. This case can also be included in Theorem 1.1 by taking $V_{t}$ to be the origin for $t \geq 0$. More general examples for $\lambda_{j}>0$, which include self-expanders with arbitrarily small Lagrangian angle, are constructed in [9].

Theorems 1.1 and 1.2 are analogous to the Feldman-Ilmanen-Knopf [2] gluing construction for the Kähler-Ricci flows. Unlike the mean curvature flow, a notion of weak solutions of Ricci flow has not yet been established.

This article is organized as follows. The Hamiltonian stationary examples in Theorem 1.1 and their geometry and topology are presented in $\S 2$. In $\S 3$, we recall the formulation of Brakke flow and prove Theorem 1.1. The proof of Theorem 1.2 and the discussion of the geometric properties of these examples are given in $\S 4$.

\section{HAMILTONIAN STATIONARY EXAMPLES}

2.1. The constructions. For any $n$ nonzero integers $\lambda_{1}, \cdots, \lambda_{n}$, consider the submanifold $L$ of $\mathbb{C}^{n}$ defined by

$$
\left\{\left(x_{1} e^{i \lambda_{1} s}, \cdots, x_{n} e^{i \lambda_{n} s}\right) \mid 0 \leq s<2 \pi, \sum_{j=1}^{n} \lambda_{j} x_{j}^{2}=C,\left(x_{1}, \cdots, x_{n}\right) \in \mathbb{R}^{n}\right\}
$$

for some constant $C$.

It is not hard to check that $L$ is Lagrangian in $\mathbb{C}^{n}$ with the Lagrangian angle given by $\theta=\left(\sum_{j=1}^{n} \lambda_{j}\right) s+\frac{\pi}{2}$. It follows that $L$ is special Lagrangian if $\sum_{j=1}^{n} \lambda_{j}=0$. In general, a direct computation shows that the induced metric on $L$ is independent of $s$. Hence $\Delta_{L} \theta=0$ and $L$ is Hamiltonian stationary.

Such special Lagrangians were studied by M. Haskins in [4, [5] (for $n=3$ ) and D. Joyce in [7] (for general dimensions). We are informed by D. Joyce that the Hamiltonian stationary examples may also be obtained by applying his method of "perpendicular symmetries" in [8].

When $C=0$, the examples are Hamiltonian stationary cones, which generalize the two-dimensional Schoen-Wolfson cones. We will study the geometry of these examples in the next subsection. Now assume the constant $C$ in the defining equation is nonzero. 
If $\sum_{j=1}^{n} \lambda_{j} \neq 0$, a direct computation shows that

$$
F^{\perp}=\frac{-C}{\sum_{j=1}^{n} \lambda_{j}} H
$$

That is, the submanifold $L$ is a Hamiltonian stationary self-similar solution of the mean curvature flow.

We summarize the calculations in this subsection in the following proposition.

Proposition 2.1. For any $n$ nonzero integers $\lambda_{1}, \cdots, \lambda_{n}$, consider the submanifold $L$ of $\mathbb{C}^{n}$ defined by

$$
\left\{\left(x_{1} e^{i \lambda_{1} s}, \cdots, x_{n} e^{i \lambda_{n} s}\right) \mid 0 \leq s<2 \pi, \sum_{j=1}^{n} \lambda_{j} x_{j}^{2}=C,\left(x_{1}, \cdots, x_{n}\right) \in \mathbb{R}^{n}\right\}
$$

for some constant $C$. It is special Lagrangian when $\sum_{j=1}^{n} \lambda_{j}=0$. If $\sum_{j=1}^{n} \lambda_{j} \neq 0$, it is Hamiltonian stationary and the normal projection of the position vector satisfies

$$
F^{\perp}=\frac{-C}{\sum_{j=1}^{n} \lambda_{j}} H
$$

Without loss of generality, we can assume that $\sum_{j=1}^{n} \lambda_{j}>0$ in this case. Then $L$ is a Hamiltonian stationary self-shrinker when $C>0$, a Hamiltonian stationary cone when $C=0$, and a Hamiltonian stationary self-expander when $C<0$. Moreover, the Hamiltonian stationary self-expander and the self-shrinker are asymptotic to the Hamiltonian stationary cone.

Remark 2.1. The same results hold for any real numbers $\lambda_{1}, \cdots, \lambda_{n}$ which are not all zeros.

2.2. The geometry of the examples. Denote

$$
F\left(x_{1}, \cdots, x_{n}, s\right)=\left(x_{1} e^{i \lambda_{1} s}, \cdots, x_{n} e^{i \lambda_{n} s}\right) .
$$

It is easy to see that

$$
F\left(x_{1}, \cdots, x_{n}, s+\pi\right)=F\left((-1)^{\lambda_{1}} x_{1}, \cdots,(-1)^{\lambda_{n}} x_{n}, s\right) .
$$

As a result, the map $F$ is generically two-to-one. To solve this problem, we restrict the domain to $0 \leq s<\pi$. The tangent planes at $F\left(x_{1}, \cdots, x_{n}, s+\pi\right)$ and $F\left((-1)^{\lambda_{1}} x_{1}, \cdots,(-1)^{\lambda_{n}} x_{n}, s\right)$ agree. They are both spanned by the vector

$$
\left(i \lambda_{1}(-1)^{\lambda_{1}} x_{1}, \cdots, i \lambda_{n}(-1)^{\lambda_{n}} x_{n}\right)
$$

and the $(n-1)$ plane in $\mathbb{R}^{n}$ which is perpendicular to the vector

$$
\left(\lambda_{1}(-1)^{\lambda_{1}} x_{1}, \cdots, \lambda_{n}(-1)^{\lambda_{n}} x_{n}\right) .
$$

Define a diffeomorphism $\psi: \mathbb{R}^{n} \rightarrow \mathbb{R}^{n}$ by

$$
\psi\left(x_{1}, \cdots, x_{n}\right)=\left((-1)^{\lambda_{1}} x_{1}, \cdots,(-1)^{\lambda_{n}} x_{n}\right)
$$

and a submanifold $\Sigma$ in $\mathbb{R}^{n}$ by

$$
\Sigma=\left\{\left(x_{1}, \cdots, x_{n}\right): \sum_{j=1}^{n} \lambda_{j} x_{j}^{2}=C\right\}
$$


The restriction of $\psi$ on $\Sigma$ is a diffeomorphism onto itself. The restriction map is an orientation-preserving map if and only if $\sum_{j=1}^{n} \lambda_{j}$ is even. It follows that for any $n$ nonzero integers $\lambda_{1}, \cdots, \lambda_{n}$, the Lagrangian submanifold $L^{\prime}$

$$
\left\{\left(x_{1} e^{i \lambda_{1} s}, \cdots, x_{n} e^{i \lambda_{n} s}\right) \mid 0 \leq s<\pi, \sum_{j=1}^{n} \lambda_{j} x_{j}^{2}=C,\left(x_{1}, \cdots, x_{n}\right) \in \mathbb{R}^{n}\right\}
$$

is oriented if and only if $\sum_{j=1}^{n} \lambda_{j}$ is even. Assume that $\lambda_{j}>0$ for $1 \leq j \leq k<n$ and $\lambda_{j}<0$ for $k+1 \leq j \leq n$. Then the ansatz of our examples can be written as

$$
\sum_{j=1}^{k}\left|\lambda_{j}\right| x_{j}^{2}=\sum_{j=k+1}^{n}\left|\lambda_{j}\right| x_{j}^{2}+C .
$$

The topology of $L^{\prime}$ is $\mathbb{R}^{k} \times S^{n-k-1} \times S^{1}$ when $C<0$, is $S^{k-1} \times \mathbb{R}^{n-k} \times S^{1}$ when $C>0$, and is a cone with link $S^{k-1} \times S^{n-k-1} \times S^{1}$, with an isolated singular point at 0 , when $C=0$. When $\lambda_{j}>0$ for all $j$, we can only consider the case $C>0$ and the topology is $S^{n-1} \times S^{1}$.

To avoid other possible self-intersections, we require that $\left|\lambda_{1}\right|, \cdots,\left|\lambda_{n}\right|$ are pairwise co-prime. This condition is sufficient to guarantee that the Hamiltonian stationary cone $(C=0)$ is embedded. In addition, we require that $\lambda_{j}=1$ for $1 \leq j \leq k$ when $C>0$ and $\lambda_{j}=-1$ for $k+1 \leq j \leq n$ when $C<0$ in order to obtain an embedded $L^{\prime}$ in each case.

Only when $C>0, k=1$, or $C<0, k=n-1$, the real hypersurface $\Sigma$ is disconnected. However, if $\lambda_{1}$ is odd in the first case, the factor $e^{i \lambda_{1} s}$ becomes -1 at $s=\pi$ and thus $L^{\prime}$ will still be connected. If $\lambda_{n}$ is odd in the second case, the Lagrangian submanifold $L^{\prime}$ will also be connected for the same reason.

We summarize these discussions in the following proposition.

Proposition 2.2. For any constant $C$ and $n$ nonzero integers $\lambda_{1}, \cdots, \lambda_{n}$, the Lagrangian submanifold $L^{\prime}$ defined by

$$
\left\{\left(x_{1} e^{i \lambda_{1} s}, \cdots, x_{n} e^{i \lambda_{n} s}\right) \mid 0 \leq s<\pi, \sum_{j=1}^{n} \lambda_{j} x_{j}^{2}=C,\left(x_{1}, \cdots, x_{n}\right) \in \mathbb{R}^{n}\right\}
$$

is oriented if and only if $\sum_{j=1}^{n} \lambda_{j}$ is even.

Assume that $\lambda_{j}>0$ for $1 \leq j \leq k$ and $\lambda_{j}<0$ for $k+1 \leq j \leq n$. The topology of $L^{\prime}$ is $\mathbb{R}^{k} \times S^{n-k-1} \times S^{1}$ when $C<0$, is $S^{k-1} \times \mathbb{R}^{n-k} \times S^{1}$ when $C>0$, and is a cone with link $S^{k-1} \times S^{n-k-1} \times S^{1}$ when $C=0$. If $C>0, k=1$ and $\lambda_{1}$ is even, or $C<0, k=n-1$ and $\lambda_{n}$ is even, there are two connected components in $L^{\prime}$. The submanifold $L^{\prime}$ is connected for all other cases.

Suppose that $\left|\lambda_{1}\right|, \cdots,\left|\lambda_{n}\right|$ are pairwise co-prime. Then the corresponding cones in the case $C=0$ are embedded. However, one also needs to require $\lambda_{j}=1$ for $1 \leq j \leq k$ to make $L^{\prime}$ embedded in the case $C>0$ and to require $\lambda_{j}=-1$ for $k+1 \leq j \leq n$ to make $L^{\prime}$ embedded in the case $C<0$.

Remark 2.2. Theorem 1.1 holds without these extra assumptions on $\lambda_{j}$.

Remark 2.3. It is worth noting that the case $\sum_{j=1}^{n} \lambda_{j}=0$ corresponds to a special Lagrangian. Hence the proposition shows that there are two families of smooth special Lagrangians which have different topologies but converge to the same special Lagrangian cone $(C=0)$. The element in one family has topology $\mathbb{R}^{k} \times S^{n-k-1} \times S^{1}$ 
(for the case $C<0$ ), and the element in the other family has topology $S^{k-1} \times \mathbb{R}^{n-k} \times$ $S^{1}$ (for the case $C>0$ ).

\section{Proof of Theorem 1.1}

Assume $\lambda_{j}$ are nonzero integers and $\sum_{j=1}^{n} \lambda_{j}>0$. Define

$$
V_{t}=\left\{\left(x_{1} e^{i \lambda_{1} s}, \cdots, x_{n} e^{i \lambda_{n} s}\right) \mid 0 \leq s<\pi, \sum_{j=1}^{n} \lambda_{j} x_{j}^{2}=(-2 t) \sum_{j=1}^{n} \lambda_{j},\left(x_{1}, \cdots, x_{n}\right) \in \mathbb{R}^{n}\right\} .
$$

The varifold $V_{t}$ for $t \neq 0$ is smooth, and $V_{0}$ is a cone with an isolated singularity at the origin. As discussed in the previous sections, $V_{t}$ are Hamiltonian stationary self-shrinkers for $t<0$ and Hamiltonian stationary self-expanders for $t>0$. As $t \rightarrow 0, V_{t}$ converges to the Hamiltonian stationary cone $V_{0}$. The geometry of $V_{t}$ is discussed in Proposition 2.2. What is left in the proof of Theorem 1.1 is to show that the varifolds $V_{t}$ for $-\infty<t<\infty$ form an eternal solution of Brakke flow without mass loss. We first recall the definition of Brakke flow.

3.1. Brakke flow. A family of varifolds $V_{t}$ is said to form a solution of the Brakke flow 1 if

$$
\bar{D}\left\|V_{t}\right\|(\phi) \leq \delta\left(V_{t}, \phi\right)\left(h\left(V_{t}\right)\right)
$$

for each $\phi \in C_{0}^{1}\left(\mathbb{R}^{n}\right)$ with $\phi \geq 0$, where $\bar{D}\left\|V_{t}\right\|(\phi)$ is the upper derivative defined by $\varlimsup_{t_{1} \rightarrow t} \frac{\left\|V_{t_{1}}\right\|(\phi)-\left\|V_{t}\right\|(\phi)}{t_{1}-t}$ and $h\left(V_{t}\right)$ is the generalized mean curvature vector of $V_{t}$. In the setting of this paper,

$$
\delta\left(V_{t}, \phi\right)\left(h\left(V_{t}\right)\right)=-\int \phi\left|h\left(V_{t}\right)\right|^{2} d\left\|V_{t}\right\|+\int D \phi \cdot h\left(V_{t}\right) d\left\|V_{t}\right\| .
$$

In our case, the family $V_{t}$ satisfy mean curvature flow for $t<0$ and $t>0$ and the singularity only happens at the $t=0$ slice. The following proposition is formulated in [10] as a criterion to check the solutions of Brakke flow in this situation.

Proposition 3.1. Suppose the varifold $V_{t}, a<t<b$, forms a smooth mean curvature flow in $\mathbb{R}^{n}$ except at $t=c \in(a, b)$ and $\left\|V_{t}\right\|$ converges in Radon measure to $\left\|V_{c}\right\|$ as $t \rightarrow c$. If $\lim _{t \rightarrow c^{-}} \frac{d}{d t}\left\|V_{t}\right\|(\phi)$ and $\lim _{t \rightarrow c^{+}} \frac{d}{d t}\left\|V_{t}\right\|(\phi)$ are both either finite or $-\infty$ and

$$
\lim _{t \rightarrow c^{ \pm}} \frac{d}{d t}\left\|V_{t}\right\|(\phi) \leq \delta\left(V_{0}, \phi\right)\left(h\left(V_{0}\right)\right)
$$

for any $\phi \in C_{0}^{1}\left(\mathbb{R}^{n}\right)$, then $V_{t}$ forms a solution of the Brakke flow.

Definition 3. If $V_{t}$ forms a solution of the Brakke flow for $-\infty<t<\infty$, we call it an eternal solution for the Brakke flow. Moreover, if the equality in (3.1) is achieved for all $-\infty<t<\infty$, we say the solution has no mass loss.

3.2. Completion of the proof. For a smooth mean curvature flow, we have

$$
\frac{d}{d t}\left\|V_{t}\right\|(\phi)=\delta\left(V_{t}, \phi\right)\left(h\left(V_{t}\right)\right)=-\int \phi\left|h\left(V_{t}\right)\right|^{2} d\left\|V_{t}\right\|+\int D \phi \cdot h\left(V_{t}\right) d\left\|V_{t}\right\| .
$$


To apply Proposition 3.1 and prove that the equality in the Brakke flow is achieved, it suffices to show that

$$
\begin{aligned}
& \lim _{t \rightarrow 0^{-}}-\int \phi\left|h\left(V_{t}\right)\right|^{2} d\left\|V_{t}\right\|+\int D \phi \cdot h\left(V_{t}\right) d\left\|V_{t}\right\| \\
& =-\int \phi\left|h\left(V_{0}\right)\right|^{2} d\left\|V_{0}\right\|+\int D \phi \cdot h\left(V_{0}\right) d\left\|V_{0}\right\|
\end{aligned}
$$

and

$$
\begin{aligned}
& \lim _{t \rightarrow 0^{+}}-\int \phi\left|h\left(V_{t}\right)\right|^{2} d\left\|V_{t}\right\|+\int D \phi \cdot h\left(V_{t}\right) d\left\|V_{t}\right\| \\
& =-\int \phi\left|h\left(V_{0}\right)\right|^{2} d\left\|V_{0}\right\|+\int D \phi \cdot h\left(V_{0}\right) d\left\|V_{0}\right\| .
\end{aligned}
$$

A direct calculation shows that

$$
\begin{aligned}
\left|V_{t}\right|^{2} & =\sum_{j=1}^{n} x_{j}^{2}, \\
\left|h\left(V_{t}\right)\right|^{2} & =\frac{\sum_{j=1}^{n} \lambda_{j}^{2}}{\sum_{j=1}^{n} \lambda_{j}^{2} x_{j}^{2}},
\end{aligned}
$$

and

$$
d\left\|V_{t}\right\|=\sqrt{\sum_{j=1}^{n} \lambda_{j}^{2} x_{j}^{2}} d S_{t} d s,
$$

where $d S_{t}$ is the volume form of the hypersurface

$$
\Sigma_{t}=\left\{\left(x_{1}, \cdots, x_{n}\right) \mid \sum_{j=1}^{n} \lambda_{j} x_{j}^{2}=(-2 t) \sum_{j=1}^{n} \lambda_{j}\right\}
$$

in $\mathbb{R}^{n}$.

We can parameterize $\Sigma_{t}$ by rewriting the defining equation as

$$
\sum_{j=1}^{k}\left|\lambda_{j}\right| x_{j}^{2}=\sum_{j=k+1}^{n}\left|\lambda_{j}\right| x_{j}^{2}-2 t \sum_{j=1}^{n} \lambda_{j},
$$

where $\lambda_{j}>0$ for $j=1 \cdots k$ and $\lambda_{j}<0$ for $j=k+1, \cdots, n$.

Suppose $X_{2}=\left(0, \cdots, 0, x_{k+1}, \cdots, x_{n}\right)$ gives the embedding of the surface

$$
\sum_{j=k+1}^{n}\left|\lambda_{j}\right| x_{j}^{2}=1
$$

and $X_{1}=\left(x_{1}, \cdots, x_{k}, 0, \cdots, 0\right)$ gives the embedding of the surface $\sum_{j=1}^{k}\left|\lambda_{j}\right| x_{j}^{2}=1$. Then the hypersurface $\Sigma_{t}$ for $t<0$ can be parameterized by

$$
X=\left(r^{2}-2 t \sum_{j=1}^{n} \lambda_{j}\right)^{\frac{1}{2}} X_{1}+r X_{2},
$$

where $r^{2}=\sum_{j=k+1}^{n}\left|\lambda_{j}\right| x_{j}^{2}$. 
It is not hard to check that the volume form of $\Sigma_{t}, t<0$, is given by (3.8)

$$
d S_{t}=r^{n-k-1}\left(r^{2}-2 t \sum_{j=1}^{n} \lambda_{j}\right)^{\frac{k-1}{2}}\left(\frac{r^{2}}{r^{2}-2 t \sum_{j=1}^{n} \lambda_{j}}\left|X_{1}^{\perp}\right|^{2}+\left|X_{2}^{\perp}\right|^{2}\right)^{\frac{1}{2}} d r d S_{t}^{-} d S_{t}^{+},
$$

where $d S_{t}^{-}$is the volume form of

$$
\left\{\left(x_{k+1}, \cdots, x_{n}\right)\left|\sum_{j=k+1}^{n}\right| \lambda_{j} \mid x_{j}^{2}=1\right\} \subset \mathbb{R}^{n-k},
$$

and $d S_{t}^{+}$is the volume form of $\left\{\left(x_{1}, \cdots, x_{k}\right)\left|\sum_{j=1}^{k}\right| \lambda_{j} \mid x_{j}^{2}=1\right\} \subset \mathbb{R}^{k}$.

From (3.5), (3.6), (3.7) and (3.8), we have

$$
\begin{aligned}
& \int \phi\left|h\left(V_{t}\right)\right|^{2} d\left\|V_{t}\right\| \\
= & \int \frac{\phi \sum_{j=1}^{n} \lambda_{j}^{2}}{\sqrt{\sum_{j=1}^{n} \lambda_{j}^{2} x_{j}^{2}}} r^{n-k-1}\left(r^{2}-2 t \sum_{j=1}^{n} \lambda_{j}\right)^{\frac{k-1}{2}} \\
& \times\left(\frac{r^{2}}{r^{2}-2 t \sum_{j=1}^{n} \lambda_{j}}\left|X_{1}^{\perp}\right|^{2}+\left|X_{2}^{\perp}\right|^{2}\right)^{\frac{1}{2}} d r d S_{t}^{-} d S_{t}^{+} d s .
\end{aligned}
$$

Because $t<0$, and $\sum_{j=1}^{n} \lambda_{j}>0$, it follows that

$$
\begin{aligned}
r^{n-k-1}\left(r^{2}-2 t \sum_{j=1}^{n} \lambda_{j}\right)^{\frac{k-1}{2}} & <\left(2 r^{2}-2 t \sum_{j=1}^{n} \lambda_{j}\right)^{\frac{n-2}{2}} \\
& =\left(\sum_{j=1}^{n}\left|\lambda_{j}\right| x_{j}^{2}\right)^{\frac{n-2}{2}} \\
& \leq\left(\sum_{j=1}^{n} \lambda_{j}^{2} x_{j}^{2}\right)^{\frac{n-2}{2}}
\end{aligned}
$$

and the integrand in (3.9) is bounded by the function

$$
\phi \sum_{j=1}^{n} \lambda_{j}^{2}\left(\sum_{j=1}^{n} \lambda_{j}^{2} x_{j}^{2}\right)^{\frac{n-3}{2}}\left(\left|X_{1}^{\perp}\right|^{2}+\left|X_{2}^{\perp}\right|^{2}\right)^{\frac{1}{2}} .
$$

Moreover, the function $\phi$ has compact support and $\left\{\lambda_{j}\right\}_{j=1}^{n}$ is fixed, so this is an integrable function when $n \geq 3$. By the dominate convergence theorem, we thus have

$$
\lim _{t \rightarrow 0^{-}}-\int \phi\left|h\left(V_{t}\right)\right|^{2} d\left\|V_{t}\right\|=-\int \phi\left|h\left(V_{0}\right)\right|^{2} d\left\|V_{0}\right\| .
$$

The same estimates also show

$$
\lim _{t \rightarrow 0^{-}} \int D \phi \cdot h\left(V_{t}\right) d\left\|V_{t}\right\|=\int D \phi \cdot h\left(V_{0}\right) d\left\|V_{0}\right\| .
$$

We thus prove (3.3). Note that when $k=1$ or $n-1$, the expression above is slightly different, but the same argument works. 
When $t>0$, we rewrite the defining equation as

$$
\sum_{j=1}^{k}\left|\lambda_{j}\right| x_{j}^{2}+2 t \sum_{j=1}^{n} \lambda_{j}=\sum_{j=k+1}^{n}\left|\lambda_{j}\right| x_{j}^{2}
$$

where $\lambda_{j}>0$ for $j=1, \cdots, k$ and $\lambda_{j}<0$ for $j=k+1, \cdots, n$. Then the hypersurface $\Sigma_{t}$ for $t>0$ can be parameterized by

$$
X=r X_{1}+\left(r^{2}+2 t \sum_{j=1}^{n} \lambda_{j}\right)^{\frac{1}{2}} X_{2}
$$

where $r^{2}=\sum_{j=1}^{k}\left|\lambda_{j}\right| x_{j}^{2}$.

Similar computations as in the case $t<0$ show that the volume form of $\Sigma_{t}$, $t>0$, is given by

$$
d S_{t}=r^{k-1}\left(r^{2}+2 t \sum_{j=1}^{n} \lambda_{j}\right)^{\frac{n-k-1}{2}}\left(\left|X_{1}^{\perp}\right|^{2}+\frac{r^{2}}{r^{2}+2 t \sum_{j=1}^{n} \lambda_{j}}\left|X_{2}^{\perp}\right|^{2}\right)^{\frac{1}{2}} d r d S_{t}^{-} d S_{t}^{+} .
$$

Therefore,

$$
\begin{aligned}
& \int \phi\left|h\left(V_{t}\right)\right|^{2} d\left\|V_{t}\right\| \\
= & \int \frac{\phi \sum_{j=1}^{n} \lambda_{j}^{2}}{\sqrt{\sum_{j=1}^{n} \lambda_{j}^{2} x_{j}^{2}}} r^{k-1}\left(r^{2}+2 t \sum_{j=1}^{n} \lambda_{j}\right)^{\frac{n-k-1}{2}} \\
& \times\left(\left|X_{1}^{\perp}\right|^{2}+\frac{r^{2}}{r^{2}+2 t \sum_{j=1}^{n} \lambda_{j}}\left|X_{2}^{\perp}\right|^{2}\right)^{\frac{1}{2}} d r d S_{t}^{-} d S_{t}^{+} d s .
\end{aligned}
$$

Because $t>0$ and $\sum_{j=1}^{n} \lambda_{j}>0$, we can similarly show that the integrand in (3.12) is bounded by the function

$$
\phi \sum_{j=1}^{n} \lambda_{j}^{2}\left(\sum_{j=1}^{n} \lambda_{j}^{2} x_{j}^{2}\right)^{\frac{n-3}{2}}\left(\left|X_{1}^{\perp}\right|^{2}+\left|X_{2}^{\perp}\right|^{2}\right)^{\frac{1}{2}}
$$

which is an integrable function if $n \geq 3$ and $\phi$ has compact support. By the dominate convergence theorem, we thus have

$$
\lim _{t \rightarrow 0^{+}}-\int \phi\left|h\left(V_{t}\right)\right|^{2} d\left\|V_{t}\right\|=-\int \phi\left|h\left(V_{0}\right)\right|^{2} d\left\|V_{0}\right\| .
$$

The same estimates also show

$$
\lim _{t \rightarrow 0^{+}} \int D \phi \cdot h\left(V_{t}\right) d\left\|V_{t}\right\|=\int D \phi \cdot h\left(V_{0}\right) d\left\|V_{0}\right\| .
$$

We thus prove (3.4). Again, when $k=1$ or $n-1$, the expression above needs slight modification, but the same argument gives the conclusion. Since the twodimensional case is already proved in [10, this completes the proof of Theorem 1.1 . 


\section{Proof of Theorem 1.2}

Recall [9, Theorem A] that if

$$
\begin{aligned}
\frac{d w_{j}}{d s} & =\lambda_{j} e^{i \theta(s)} \overline{w_{1} \cdots w_{j-1} w_{j+1} \cdots w_{n}}, \quad j=1, \cdots, n, \\
\frac{d \theta}{d s} & =\alpha \operatorname{Im}\left(e^{-i \theta(s)} w_{1} \cdots w_{n}\right),
\end{aligned}
$$

then the submanifold $L$ in $\mathbb{C}^{n}$ given by

$$
L=\left\{\left(x_{1} w_{1}(s), \cdots, x_{n} w_{n}(s)\right): s \in I, x_{1}, \cdots, x_{n} \in \mathbb{R}, \sum_{j=1}^{n} \lambda_{j} x_{j}^{2}=C\right\}
$$

is Lagrangian, with Lagrangian angle $\theta(s)$ at $\left(x_{1} w_{1}(s), \cdots, x_{n} w_{n}(s)\right)$, and its position vector $F$ and mean curvature vector $H$ satisfy $\alpha F^{\perp}=C H$.

Because there is a lot of freedom to rescale the constants (see [9, Remark 3.2]), we can assume $\alpha=1$ for simplicity. From Theorem $\mathrm{F}$ of $[9]$, there is a dense set of initial data such that the solutions $w_{1}(s), \cdots, w_{n}(s)$ and $\theta(s)$ of (4.1) are periodic. Suppose the period is $T$. Then $V_{t}$, which is defined by

$$
V_{t}=\left\{\left(x_{1} w_{1}(s), \cdots, x_{n} w_{n}(s)\right): 0 \leq s<T, \sum_{j=1}^{n} \lambda_{j} x_{j}^{2}=2 t,\left(x_{1}, \cdots, x_{n}\right) \in \mathbb{R}^{n}\right\},
$$

is a Lagrangian self-shrinker for $t<0$, a Lagrangian self-expander for $t>0$, and a Lagrangian cone for $t=0$.

To show this family forms an eternal solution of Brakke flow without mass loss, as in the proof of Theorem 1.1. it remains to check (3.3) and (3.4).

Denote $w_{j}(s)=r_{j}(s) e^{i \varphi_{j}(s)}$ and $\varphi(s)=\sum_{j=1}^{n} \varphi_{j}(s)$, where $r_{j}(s)=\left|w_{j}(s)\right|$. Remember that $r_{j}$ has positive lower and upper bounds. A direct calculation shows that

$$
\begin{gathered}
\left|V_{t}\right|^{2}=\sum_{j=1}^{n} r_{j}^{2} x_{j}^{2}, \\
\left|h\left(V_{t}\right)\right|^{2}=\left(\sum_{j=1}^{n} \frac{\lambda_{j}^{2} x_{j}^{2}}{r_{j}^{2}}\right)^{-1} \sin ^{2}(\varphi-\theta),
\end{gathered}
$$

and

$$
d\left\|V_{t}\right\|=\frac{r_{1}^{2} \cdots r_{n}^{2}}{\sqrt{\sum_{j=1}^{n} \lambda_{j}^{2} x_{j}^{2}}} \sum_{j=1}^{n} \frac{\lambda_{j}^{2} x_{j}^{2}}{r_{j}^{2}} d S_{t} d s
$$

where $d S_{t}$ is the volume form of the hypersurface

$$
\Sigma_{t}=\left\{\left(x_{1}, \cdots, x_{n}\right) \mid \sum_{j=1}^{n} \lambda_{j} x_{j}^{2}=2 t\right\}
$$

in $\mathbb{R}^{n}$. When $t<0$, we can parameterize $\Sigma_{t}$ by rewriting the defining equation as

$$
\sum_{j=1}^{k}\left|\lambda_{j}\right| x_{j}^{2}-2 t=\sum_{j=k+1}^{n}\left|\lambda_{j}\right| x_{j}^{2}
$$

Suppose $X_{1}=\left(x_{1}, \cdots, x_{k}, 0, \cdots, 0\right)$ gives the embedding of the surface $\sum_{j=1}^{k}\left|\lambda_{j}\right| x_{j}^{2}$ $=1$ and $X_{2}=\left(0, \cdots, 0, x_{k+1}, \cdots, x_{n}\right)$ gives the embedding of the surface 
$\sum_{j=k+1}^{n}\left|\lambda_{j}\right| x_{j}^{2}=1$. Then the hypersurface $\Sigma_{t}$ for $t<0$ can be parameterized by

$$
X=r X_{1}+\left(r^{2}-2 t\right)^{\frac{1}{2}} X_{2},
$$

where $r^{2}=\sum_{j=1}^{k}\left|\lambda_{j}\right| x_{j}^{2}$.

It is not hard to check that the volume form of $\Sigma_{t}, t<0$, is given by

$$
d S_{t}=r^{k-1}\left(r^{2}-2 t\right)^{\frac{n-k-1}{2}}\left(\left|X_{1}^{\perp}\right|^{2}+\frac{r^{2}}{r^{2}-2 t}\left|X_{2}^{\perp}\right|^{2}\right)^{\frac{1}{2}} d r d S_{t}^{-} d S_{t}^{+}
$$

where $d S_{t}^{-}$is the volume form of

$$
\left\{\left(x_{k+1}, \cdots, x_{n}\right)\left|\sum_{j=k+1}^{n}\right| \lambda_{j} \mid x_{j}^{2}=1\right\} \subset \mathbb{R}^{n-k}
$$

and $d S_{t}^{+}$is the volume form of $\left\{\left(x_{1}, \cdots, x_{k}\right)\left|\sum_{j=1}^{k}\right| \lambda_{j} \mid x_{j}^{2}=1\right\} \subset \mathbb{R}^{k}$.

From (4.2), (4.3), (4.4) and (4.5), we have

$$
\begin{aligned}
& \int \phi\left|h\left(V_{t}\right)\right|^{2} d\left\|V_{t}\right\| \\
= & \int \frac{\phi \sin ^{2}(\varphi-\theta) r_{1}^{2} \cdots r_{n}^{2}}{\sqrt{\sum_{j=1}^{n} \lambda_{j}^{2} x_{j}^{2}}} r^{k-1}\left(r^{2}-2 t\right)^{\frac{n-k-1}{2}} \\
& \times\left(\left|X_{1}^{\perp}\right|^{2}+\frac{r^{2}}{r^{2}-2 t}\left|X_{2}^{\perp}\right|^{2}\right)^{\frac{1}{2}} d r d S_{t}^{-} d S_{t}^{+} d s .
\end{aligned}
$$

Similar to (3.10), we have

$$
r^{k-1}\left(r^{2}-2 t\right)^{\frac{n-k-1}{2}}<\left(\sum_{j=1}^{n}\left|\lambda_{j}\right| x_{j}^{2}\right)^{\frac{n-2}{2}} \leq\left(\sum_{j=1}^{n} \lambda_{j}^{2} x_{j}^{2}\right)^{\frac{n-2}{2}} .
$$

Here for simplicity we use the normalization in [9, Remark 3.2] and assume $\left|\lambda_{j}\right| \geq 1$ for all $j$. Because $\phi$ is a $C^{1}$ function with compact support, $t<0$, and $r_{1}, \cdots, r_{n}$ are bounded, the integrand in (4.6) is bounded by the function

$$
C\left(\sum_{j=1}^{n} \lambda_{j}^{2} x_{j}^{2}\right)^{\frac{n-3}{2}}\left(\left|X_{1}^{\perp}\right|^{2}+\left|X_{2}^{\perp}\right|^{2}\right)^{\frac{1}{2}} .
$$

This is an integrable function when $n \geq 3$. By the dominate convergence theorem, we thus have

$$
\lim _{t \rightarrow 0^{-}}-\int \phi\left|h\left(V_{t}\right)\right|^{2} d\left\|V_{t}\right\|=-\int \phi\left|h\left(V_{0}\right)\right|^{2} d\left\|V_{0}\right\| .
$$

The same estimates also show

$$
\lim _{t \rightarrow 0^{-}} \int D \phi \cdot h\left(V_{t}\right) d\left\|V_{t}\right\|=\int D \phi \cdot h\left(V_{0}\right) d\left\|V_{0}\right\| .
$$

We thus prove (3.3) when $n \geq 3$. Again, when $k=1$ or $n-1$, the expression above needs slight modification, but the same argument works.

When $n=2$, a direct computation gives

$$
\int \phi\left|h\left(V_{t}\right)\right|^{2} d\left\|V_{t}\right\|=\int \frac{\phi \sin ^{2}(\varphi-\theta) r_{1}^{2} r_{2}^{2}}{\sqrt{\sum_{j=1}^{2} \lambda_{j}^{2} x_{j}^{2}}}\left(1+\frac{x_{1}^{2}}{\left|\lambda_{2}\right| x_{2}^{2}}\right)^{\frac{1}{2}} d x_{1} d s
$$


where $\left|\lambda_{1}\right| x_{1}^{2}-2 t=\left|\lambda_{2}\right| x_{2}^{2}$. We proceed as in [10] by dividing it into two cases, $\phi(0)=0$ or $\phi(0) \neq 0$. When $\phi(0)=0$, we have $\phi\left(V_{t}\right) \leq C\left|V_{t}\right| \leq C^{\prime}\left(\sum_{j=1}^{2} \lambda_{j}^{2} x_{j}^{2}\right)^{\frac{1}{2}}$. With this extra power, the integrand becomes bounded and (3.3) follows from the dominate convergence theorem.

If $\phi(0) \neq 0$, we have $\int \phi\left|h\left(V_{0}\right)\right|^{2} d\left\|V_{0}\right\|=\infty$. We can also prove that

$$
\lim _{t \rightarrow 0^{-}} \int \phi\left|h\left(V_{t}\right)\right|^{2} d\left\|V_{t}\right\|=\infty
$$

and that both $\lim _{t \rightarrow 0^{-}} \int D \phi \cdot h\left(V_{t}\right) d\left\|V_{t}\right\|$ and $\int D \phi \cdot h\left(V_{0}\right) d\left\|V_{0}\right\|$ are finite. Hence (3.3) holds trivially. Now we give the proof for these facts. Given any $\epsilon>0$, there exist a $\delta>0$ such that $|\phi| \geq \epsilon$ in $B_{\delta}(0)$. Using the normalization in [9, Remark 3.2], we can assume $\lambda_{1}=1$ and $\lambda_{2}=-1$ for simplicity. Thus $x_{1}^{2}-2 t=x_{2}^{2}$ and, because $r_{1}, r_{2}$ and $\sin ^{2}(\varphi-\theta)$ all have positive lower bound, from (4.8) and $0 \leq s \leq T$ we have

$$
\int \phi\left|h\left(V_{t}\right)\right|^{2} d\left\|V_{t}\right\| \geq C \epsilon \int_{0}^{a} \frac{1}{\sqrt{2 x_{1}^{2}-2 t}} d x_{1} .
$$

Note that $\left|V_{t}\right|^{2}=x_{1}^{2} r_{1}^{2}+x_{2}^{2} r_{2}^{2} \leq \delta^{2}$ implies $x_{1}^{2}\left(r_{1}^{2}+r_{2}^{2}\right)-2 t r_{2}^{2} \leq \delta^{2}$. Hence when $t$ is close to $0^{-}$, this set contains a uniform interval $[0, a]$ where $a$ depends only on $\delta$. Since $\sqrt{2 x_{1}^{2}-2 t} \leq \sqrt{2}\left|x_{1}\right|+\sqrt{2} \sqrt{-t}$, (4.9) becomes

$$
\int \phi\left|h\left(V_{t}\right)\right|^{2} d\left\|V_{t}\right\| \geq C^{\prime} \epsilon \ln \frac{a+\sqrt{-t}}{\sqrt{-t}},
$$

which tends to $\infty$ as $t$ tends to $0^{-}$. A direct computation gives

$$
\int D \phi \cdot h\left(V_{t}\right) d\left\|V_{t}\right\| \leq \int \frac{|D \phi| r_{1}^{2} r_{2}^{2}}{\sqrt{2 x_{1}^{2}-2 t}} \sqrt{\frac{x_{1}^{2}}{r_{l}^{2}}+\frac{x_{1}^{2}-2 t}{r_{2}^{2}}}\left(1+\frac{x_{1}^{2}}{x_{1}^{2}-2 t}\right)^{\frac{1}{2}} d x_{1} d s .
$$

The integrand is bounded, and hence we can use the dominate convergence theorem to show that the limit is finite. This proves (3.3) when $n=2$.

When $t>0$, similar arguments give (3.4) for $n \geq 3$ and $n=2$. Thus Theorem 1.2 is proved.

Remark 4.1. Similar to the discussions in $\S 2.2$ (and also see [9, Theorem A and Theorem $\mathrm{F}]$ ), $V_{t}$ is a closed, nonsingular, immersed Lagrangian self-expander in $\mathbb{C}^{n}$ diffeomorphic to $S^{k-1} \times \mathbb{R}^{n-k} \times S^{1}$ when $t>0$, and a closed, nonsingular, immersed Lagrangian self-shrinker in $\mathbb{C}^{n}$ diffeomorphic to $\mathbb{R}^{k} \times S^{n-k-1} \times S^{1}$ when $t<0$, and $V_{0}$ is a closed, immersed Lagrangian cone in $\mathbb{C}^{n}$ with link $S^{k-1} \times S^{n-k-1} \times S^{1}$, with an isolated singular point at 0 . To study the embeddedness of these examples, one needs to have a better understanding of $\gamma_{j}$, which is defined in [9, Theorem E].

\section{REFERENCES}

1. K.A. Brakke, The motion of a surface by its mean curvature. Mathematical Notes, Princeton University Press, 1978. MR485012 (82c:49035)

2. M. Feldman; T. Ilmanen; D. Knopf, Rotationally symmetric shrinking and expanding gradient Kähler-Ricci solitons. J. Differential Geom. 65 (2003), no. 2, 169-209. MR 2058261 (2005e:53102)

3. R. Harvey; H.B. Lawson, Calibrated geometries. Acta Math. 148 (1982), 48-156. MR666108 (85i:53058)

4. M. Haskins, Special Lagrangian cones. Amer. J. Math. 126 (2004), no. 4, 845-871. MR2075484 (2005e:53074) 
5. M. Haskins, The geometric complexity of special Lagrangian $T^{2}$-cones. Invent. Math. 157 (2004), no. 1, 11-70. MR 2135184 (2005m:53085)

6. G. Huisken, Asymptotic behavior for singularities of the mean curvature flow. J. Differential Geom. 31 (1990), no. 1, 285-299. MR1030675 (90m:53016)

7. D. Joyce, Constructing special Lagrangian $m$-folds in $\mathbb{C}^{m}$ by evolving quadrics. Math. Ann. 320 (2001), no. 4, 757-797. MR1857138(2002j:53066)

8. D. Joyce, Special Lagrangian $m$-folds in $\mathbb{C}^{m}$ with symmetries. Duke Math. J. 115 (2002), no. 1, 1-51. MR 1932324 (2003m:53083)

9. D.D. Joyce; Y.I. Lee; M.P. Tsui, Self-similar solutions and translating solutions for Lagrangian mean curvature flow. arXiv: math.DG/0801.3721.

10. Y.I. Lee; M.T. Wang, Hamiltonian stationary shrinkers and expanders for Lagrangian mean curvature flows. To appear in J. Differential Geom., also available at arXiv: math.DG/0707.0239.

11. K. Smoczyk, Angle theorems for the Lagrangian mean curvature flow. Math. Z. 240 (2002), no. 4, 849-883. MR.1922733 (2003g:53120)

12. K. Smoczyk; M.-T. Wang, Mean curvature flows of Lagrangian submanifolds with convex potentials. J. Differential Geom. 62 (2002), no. 2, 243-257. MR.1988504 (2004d:53086)

13. R. Schoen; J.G. Wolfson Minimizing area among Lagrangian surfaces: The mapping problem. J. Differential Geom. 58 (2001), no. 1, 1-86. MR.1895348 (2003c:53119)

14. A. Strominger; S.-T. Yau; E. Zaslow, Mirror symmetry is T-duality. Nuclear Phys. B 479 (1996), no. 1-2, 243-259. MR1429831 (97j:32022)

15. M.-T. Wang, Deforming area preserving diffeomorphism of surfaces by mean curvature flow. Math. Res. Lett. 8 (2001), no. 5-6, 651-662. MR1879809 (2003f:53122)

16. M.-T. Wang, Long-time existence and convergence of graphic mean curvature flow in arbitrary codimension. Invent. Math. 148 (2002), no. 3, 525-543. MR 1908059 (2003b:53073)

17. J.G. Wolfson, Lagrangian homology classes without regular minimizers. J. Differential Geom. 71 (2005), 307-313. MR2197143(2006j:53119)

Department of Mathematics and Taida Institute for Mathematical Sciences, National Taiwan University, Taipei, Taiwan - and - National Center for Theoretical Sciences, Taipei Office, Old Mathematics Building, National Taiwan University, Taipei 10617, TAIWAN

E-mail address: yilee@math.ntu.edu.tw

Department of Mathematics, Columbia University, New York, New York 10027

E-mail address: mtwang@math.columbia.edu 